\title{
Molecular Characterization of CTX-M15 Beta Lactamase Producing Escherichia coli Isolates in Intensive Care Units
}

\author{
Rehab M. Ateya ${ }^{1 *}$, Ghada E. Amr ${ }^{1}$, Hanan E. Mohamed ${ }^{1}$ and Neveen M. Gamil ${ }^{2}$ \\ ${ }^{1}$ Departments of Clinical Pathology, Faculty of Medicine, Zagazig University, Egypt \\ ${ }^{2}$ Anaesthesia and Intensive Care, Faculty of Medicine, Zagazig University, Egypt \\ *Corresponding author
}

\section{Keywords}

CTX-M15, ICUs, E. coli, PCR, ESBL, Sequencing

Article Info

Accepted:

04 May 2019

Available Online:

10 June 2019

\section{A B S T R A C T}

The rapid emergence of multiresistant microbial pathogens is a serious threat to human health. Extended spectrum beta lactamase (ESBL)-producing Escherichia coli is a superbug causing worldwide outbreaks, CTX-M has become the most commonly detected ESBL genotype; these enzymes have become predominant worldwide, the most common type of CTX-M reported in various geographic regions is CTX-M15. A total of 80 Escherichia coli $(E$. coli) isolates were recovered from different clinical samples of intensive care units (ICUs) patients. These isolates were identified by Matrix-assisted laser desorption ionization-time of flight mass spectrometry (MALDI-TOF MS), in vitro antimicrobial susceptibility was investigated by VITEK 2 instrument, ESBL production was assessed phenotypically and ESBL genes (TEM, SHV and CTX-M15) confirmed genotypically by PCR and further confirmation for CTX-M15 by DNA sequencing. More than $50 \%$ of ESBL E. coli isolates were multidrug resistant; while carbapenams remains the most active compound among the studied isolates. Among ESBL E. coli, CTX-M-15 was $90 \%$ which was confirmed by sequencing. To our knowledge, this is the first report to study CTX-M-15 in E. coli isolates in Sharkia governorate. This study showed a high rate of ESBL E. coli with the widespread dissemination of CTX-M-15 which emphasize the need for employing an excellent management program in antibiotic therapy.

\section{Introduction}

ESBLs are beta-lactamase group of enzymes that break down antibiotics belonging to the penicillin and extended-spectrum/third generation cephalosporins (e.g., ceftriaxone, cefotaxime and/or ceftazidime) and render them ineffective. ESBL has generally been defined as transmissible beta lactamases that can be inhibited by clavulanic acid, tazobactam or sulbactam (Bouxom et al., 2018).
ESBLs are of great concern because they are often plasmid-associated that carry antimicrobial resistance genes for other antibiotics which results in treatment problems, higher morbidity, mortality, and increased health care costs (Zhang et al., 2019).

More than 125 CTX-M enzymes are currently known. Despite their name, a few are more active on ceftazidime than cefotaxime. They have mainly been found in strains of 
Salmonella enterica serovar Typhimurium and $E$. coli, but have also been described in other species of enterobacteriaceae, currently CTX-M-15 is the most widespread type in $E$. coli (Bonnedahl et al., 2014).

While E. coli was the first species in which the CTX-M type ESBLs was reported as early as 1990. Now, the CTX-M phenotype has also been reported in E. coli strains isolated from healthy humans, livestock, companion animals, food products, and sewage indicating a large scale of the reservoirs harboring and disseminating these ESBLs (Melo et al., 2018).

Infections with ESBL E coli have been linked with poorer clinical outcomes and prolonged hospitalization on average compared to infections. This is likely exacerbated in resource-limited settings, where access to newer antibiotics is often limited by cost (Maina et al., 2017).

The aim of this study was to characterize CTX-M15 beta lactamase producing E. coli isolates as a cause of infections in Zagazig university hospitals ICUs and to study its incidence, antimicrobial resistant profile and to investigate the presence of ESBL genes as a cause of antimicrobial resistance among the studied isolates.

\section{Materials and Methods}

This study is descriptive cross-sectional study, a total of $80 \mathrm{E}$. coli isolates were recovered from patients admitted to the ICUs of Zagazig University Hospitals over 6 months period from June 2016 to December 2017. Approval for this study was obtained from Research Administration and Research Ethics Committee of Faculty of Medicine, Zagazig University; the samples were sputum, urine, blood, pus, and body fluid. The collected samples were transported to the microbiology laboratory and inoculated on suitable media that incubated at $37^{\circ} \mathrm{C}$ for $24-48$ hours.

\section{Identification}

All the isolates were subjected to Gram stain then identified by (MALDI-TOF MS) using the VITEK MS system (Biomerieux) small amount of freshly grown $E$. coli isolates were picked and smeared on the wells then overlaid with $1 \mu \mathrm{L}$ of cyano hydroxy cinnamic acid (CHCA) matrix solution and allowed to dry before sent to acquisition station of MALDITOF MS. E. coli isolates were further investigated by:

\section{Antibiotic susceptibility testing}

Antibiotic susceptibility testing was carried out using Vitek 2 System (card no 71) for Gram negative bacilli (Biomerieux) in accordance with the guidelines of the Clinical and Laboratory Standards Institute (CLSI, 2018), the following antibiotics were included: Ampicillin, Amoxyclav, Trimethoprim/sulfamethoxazole (SXT), Amikacin, Imipenem, Meropenem, Tobramycin, Azetronam, Cefipeme Ceftazidime, Cefotaxime, Ceftriaxone Piperacillin/Tazobactam, Gentamicin, Ciprofloxacin, Norfloxacin, Tigecycline Nitrofurantoin, Ampicillin/Sulbactam

\section{ESBL production detection}

ESBL production was detected using Phenotypic screening test by Kirby -Bauer disk diffusion method using Mueller-Hinton agar, according to manufacture structure and to the CLSI criteria (CLSI, 2018), ESBL phenotypes confirmed by the double-disk synergy (DDS) test that carried out with amoxicillin-clavulanat $(20+10 \mu \mathrm{g})$, cefotaxime $(30 \mu \mathrm{g})$, ceftazidime $(30 \mu \mathrm{g})$ Oxoid, ESBL E test strip (BioMérieux SA, France) CT/CTL and TZ/TZL and Vitek2 system. 


\section{PCR}

All ESBL E. coli isolates were analyzed by PCR to detect TEM, SHV and CTX-M15 genes as follow:

DNA was extracted from isolated E. coli colonies by using QIAamp®DNA Mini kit QIAGEN), for DNA amplification PCR was perfermed using thermal cycler (Gene Amp PCR system 2400, Roche and ready to use (Taq PCR Master Mix Kit QIAGEN, GERMANY) were used as described by the manufacture. PCR amplification conditions for CTXM-15 were as follows: with cycling condition of initial denaturation step at $94^{\circ} \mathrm{C}$ for $2 \mathrm{~min}$ followed by 35 cycles of Denaturation at $95^{\circ} \mathrm{c}$ for $20 \mathrm{sec}$. Annealing at $55^{\circ} \mathrm{c}$ for $90 \mathrm{sec}$. Extension at $72{ }^{\circ} \mathrm{c}$ for $90 \mathrm{sec}$ and final extension at $72{ }^{\circ} \mathrm{c}$ for $10 \mathrm{~min}$. The amplified PCR products were visualized on 2 $\%$ agarose gel stained with ethidium bromide and examined under ultraviolet light; the molecular size marker is Sizer TM-50 Plus DNA Marker Solution iNtRON, A single DNA band at $840 \mathrm{bp}$ bp was recorded as positive for the TEM gene and at $776 \mathrm{bp} b p$ was recorded as positive for the SHV gene, for $C T X-M 15$ bands were expected to be at 995 bp Primers used in this study are shown on table 1

\section{Sequencing of CTX-M15}

Amplified DNAs of CTX-M15 genes were sequenced by an automated sequencing analyzer (3500xL Genetic Analyzer Applied Biosystem, USA). Sequence data were analyzed using the sequence software alignment and were compared to the identified CTX-M15 gene in the GenBank nucleotide database available on the Internet at the National center of biotechnology information website (http://www.ncbi.nlm. nih.gov) (NCBI), and nucleotide BLAST (Basic Local Alignment Search Tool) web site for sequencing analysis services and for detection of CTXM15 gene.

\section{Statistical analysis}

Data were analyzed using SPSS 20. Chi Square was used to compare categorical variables. $\mathrm{P}$ value of 0.05 was considered statistically significant

\section{Results and Discussion}

There were 70 out of $80 \mathrm{E}$. coli isolates ESBL positive by screening test; the highest percentage of ESBL producing $E$. coli was found in internal medicine ICU (38/70) 98\%, followed by anathestic ICU (16/70) $83 \%$. The most frequent ESBL positive E. coli isolated from urine with high percentage 35/70 (95\%) followed by blood 14/70(81\%).The majority of ESBL positive strains were community acquired $(38 \%)$, while hospital acquired infection were (32\%).

\section{Antimicrobial susceptibility}

There is significant difference in resistant pattern between ESBL positive and ESBL negative E. coli isolates to: Ampicillin, SXT, Cefipeme, Azetronam, Ceftazidime, Cefotaxime, Piperacillin/Tazobactam Ceftriaxone, Gentamicin, Ciprofloxacin and Ampicillin/Sulbactam, drug resistance pattern of ESBL positive and ESBL negative isolates are illustrated in (Figure 1). Carbapenemes was the most active antibiotic against ESBL E. coli (sensitivity was 97\%). In CTX-M15 positive isolates there was significant difference in resistance to Gentamicin in relation to other ESBL producing isolates.

\section{ESBL production}

Evaluation of ESBL confirmatory test in comparison with PCR for detection of CTXM15 gene (Figure 2), the highest sensitivity 
was obtained with vitek2, while the highest specificity was recorded by Etest (TZL) (Figure 3), and by using DDST (CAZ) sensitivity was $82.5 \%$ and $86 \%$ for specificity.

Results of Genetic Analysis by PCR the most predominant gene is CTXM-15(60\%) figure (4) followed by combination of CTXM-15 and TEM (20\%), CTXM-15 and SHV (7.10\%) then TEM alone (5.70\%), SHV alone $(4.30 \%)$ and least percent $(2.9 \%)$ for combination of CTXM-15 + SHV+TEM (Figure 5). There is statistically significant difference for the distribution of different ESBL genotypes E. coli producers, CTX-M15 alone is $63.1 \%$ among community acquired infection comparing to $56.3 \%$ hospital acquired infection, the combination of CTXM15 +TEM is $23.7 \%$ hospital acquired infection and $15.6 \%$ community acquired. There is difference in distribution of ESBL genotypes of studied E. coli isolates among clinical specimens, in urine specimens CTXM15 alone found with high percentage $(51.3 \%)$ followed by CTXM-15+TEM $(22.9 \%)$ there is statistically insignificant difference between ESBL genotypes of studied E. coli isolates regarding sex of patients.

\section{Sequencing of CTX-M15}

Sequencing of the CTX-M15 PCR products for two tested isolates. The results of sequencing matched CTX-M15 in all the isolates,

The sequence obtained was submitted to Gen Bank under SCEC020026, REP215 and MS05 strains with accession numbers CP034956, MG844172 and JQ397663.1 and DQ485310.1 respectively. the results revealed high degree of similarity between query (sequenced genes) and subject gene (reference genes) of NCBI- BLAST, that could be demonstrated by the high percentage of identity, which range between 98- 99\% these results illustrated in (Table 2).

Antimicrobial resistance is indiscriminate and growing problem across different bacterial groups and against all antibiotic classes, impacting every region and country in the world (Yasir et al., 2018).

Antimicrobial resistance in E. coli has gained much attention since resistance against betalactam antibiotics are increasingly documented, and several studies reported the increase of $E$. coli resistance to more than one drug or class of drugs (Tadesse et al., 2012).

Early identification of ESBL in due time is mandatory not only for optimal patient management but also for immediate establishment of appropriate infection control measures to prevent the spread of these pathogens and also to prevent hospital acquired infections and outbreaks in the community (Lim et al., 2019).

CTX-M has become the most commonly detected ESBL genotype. Because CTX-M family genes are present on plasmids or other mobile genetic elements, these enzymes have become predominant worldwide and are substituting for the more common SHV and TEM, the most common type of CTX-M reported in various geographic regions is CTX-M15; these strains are usually MDR and are detected not only in nosocomial infections but also in the community (Hashemizadeh et al., 2018).

In agreement with global reports, an alarming increase in resistance to the extendedspectrum subclass, among clinical E. coli isolates is highlighted by the results of this study. We found that 70 out of 80 screened $\mathrm{E}$ coli isolates $(87.5 \%)$ gave positive results. In Egypt these results were in agreement with 
Ramadan et al., (2019) who reported (90.4\%) and in partial agreement with Abdel-Moaty et al., (2016) and Fam and El-Damarawy (2008) which was carried out in Intensive Care Unit. On the contrary, our prevalence rate was higher than that recorded by Abdallah et al., (2015), Fadil et al., (2017) and Khater and Sherif (2014) where prevalence rate was $54.5 \%, 61.1 \%$ and $53.3 \%$ respectively. Our results showed a high prevalence rate compared to many European countries according to (antimicrobial resistance/ database/Pages/database.aspx)

This discrepancy may be attributed to the geographical distribution, selection criteria and sample size of screened population where some studies conducted on specific groups of patients e.g. blood stream infection by Abdallah et al., (2015).

Regarding gender, our study showed that there was no statistically significant difference between males (83\%) and females (91\%) regarding frequency of ESBL positive strains.

In agreement with Akanbi et al., (2013) who reported that no differences were apparent between ESBL-producing $E$. coli with gender distribution, but on the other hand Fody et al., (2017) had shown that females had a higher prevalence rate than males.

In this study the distribution of suspected ESBL positive $E$. coli strain was highest in urine $(95 \%)$, followed by blood $(81 \%)$ and this agreed with Maina et al., (2017), Abdallah et al., (2015) and Raut et al., (2015).

On the other hand, some studies detected a high rate of ESBL-producing E. coli in blood as Zhang et al., (2014) and Fody et al., (2017), while Fadil et al., (2017) reported that suspected ESBL positive E. coli strain from sputum was higher than in other samples.
The frequency distribution of ESBL detected by DDST-CAZ positive results was higher in this study than that detected by DDST -CTX, these results are similar to Sujatha et al., (2017). Many studies agreed with our study in reporting the high sensitivity of VITEK 2 in relation to the specificity as Kumar et al., (2018), Liu et al., (2015) and El-Jade et al., (2016).

Phenotypic testing of ESBL $E$. coli by Etest when using PCR as the reference method, our results indicate that Etest TZL is characterized by high sensitivity and specificity, this interpretation is reflected clearly in a study laid down by Kałużn et al., (2014) particularly with regard to E. coli.

This difference may reflect the different types of ESBLs in our isolates, and emphasizes the importance of testing more than one cephalosporin.

In the present study, Molecular characterization revealed high frequency of CTX-M15 containing isolates and this agreed with previous reports on CTX-M15 carried in Egypt as Ramadan et al., (2019), Abdallah et al., (2015) and Khalaf et al., (2009).

Yasir et al., (2018), Guiral et al., (2018) and Maina et al., (2017) support our results as high rate of CTX-M 15 E. coli, while SHV and TEM had lower percentage.

CTX-M15 has shown to be a continuously increasing problem in several countries; Germany, Cameroon, Saudi-Arabia, and Lebanon, CTX-M-15 is considered the most frequent ESBL (Pietsch et al., 2015; Valenza et al., 2015; Djuikoue et al., 2017; Mashwal et al., 2017; Al-Agamy et al., 2014).

The high prevalence of CTX-M15 worldwide could be attributed to the successful dissemination of genetic elements and the 
clonal expansion of E. coli (Rogers et al., 2011). Apart from the environment animals that are a food source serve as reservoirs for CTX-M15 spread (Ali et al., 2016), also the powerful ability of CTX-M15 gene products to hydrolyze ceftazidime, cefotaxime and aztreonam, which probably offers a selective pressure on the bacteria especially when multiple antibiotics are concomitantly or consecutively prescribed (Ramadan et al., 2019).

In contrast to our results, low prevalence rate of CTX-M15 was observed in the study conducted by Dasgupta et al., (2018) and Bajpai et al., (2017) revealed that the gene predominated was TEM followed by CTXM15 and SHV. Also, all ESBL producing $E$. coli strains were CTX-M 15, while no strain of E. coli had TEM or SHV as shown by Djuikoue et al., (2017).

This difference may be attributed to the variation in the frequency and predominant type of ESBL genes from region to region and even between institutions within the same region.

In the present study percentage of ESBL positive strain of studied $E$. coli isolates regarding department of ICUs was highly significant in internal medicine ICU was $98 \%$ which is alarming, this finding is in agreement with Chakraborty et al., (2013).

There was statistically significant difference for the distribution of CTX-M15; in our study it was $63.1 \%$ among community acquired infection compared to $56.3 \%$ hospital acquired infection (ICUs). Implying the increased spread in the community, in concordance with our results, Abrar et al., (2017); one of Indian study Chakraborty et al., (2013) and Yasir et al., (2018) reported that the majority of the ESBL-positive E. coli CTXM-15 were of community-onset.
Among 19 antibiotics tested in this study, E. coli ESBL isolates have high percentage of multidrug resistance while ESBL positive $E$. coli isolates were still sensitive to Imipenem, Meropenem however, it will not be long until extended exposure to carbapenams will have its impact on selecting for bacteria that are resistant to this last-resort antibiotic (Li et al., 2018) have already described the emergence of carbapenem resistance in Enterobacteriaceae

Our findings are in agreement with Ouedraogo et al., (2015) showed a susceptibility of $100 \%$ for imipenem and Mohajeri et al., (2014) reported high resistance to various antimicrobial classes

The high susceptibility of ESBL-producing bacteria to carbapenems may be attributed to the fact that carbapenems are highly stable to ESBL hydrolytic activity in addition their penetration to the outer bacterial membrane is excellent due to their compact molecular size (Harada et al., 2008).

There were $(48 \%)$ of the CTX-M15 producing isolates resistant to Gentamicin which is similar to reports from other studies Kanamori et al., (2011); Strahilevitz et al., (2009) and Fallah et al., (2016).

Molecular characterization of isolates encoding CTX-M15 from $E$. coli involved in outbreaks in different countries has demonstrated that they additionally carried other antibiotic resistance genes Fallah et al., (2016).

The results of genotyping tests showed that 63 isolates which had been detected for the prevalence of CTX-M15 genes by PCR, all are matched by sequencing technique as CTX-M15 in all the isolates the results showed high identity (98-100\%) with reference protein of gene bank with CTXM15 of NCBI-BLAST. 
These results were in agreement with Wickramasinghe et al., (2012) for Accession NO. (DQ485310.1). Similarly, our results agreed with results obtained by Lopes et al.,
(2017), Tacão et al., (2012) for Accession NO (MG844172.1) and Accession NO (JQ397663.1) respectively, as concerning protein sequence.

Table.1 Sequences of primers used in PCR

\begin{tabular}{|c|c|c|c|c|}
\hline Gene & Primer & Sequence (5' to $\left.3^{\prime}\right)$ & Amplicon size (bp) & Ref \\
\hline \multirow[b]{2}{*}{ CTXM15 } & Ctxm15F & $\begin{array}{r}\text { CAC ACG TGG AAT } \\
\text { TTA GGG ACT }\end{array}$ & \multirow[b]{2}{*}{995 bp } & \multirow[t]{2}{*}{$\begin{array}{r}\text { Muzaheed } \\
\text { et al.,2008 }\end{array}$} \\
\hline & CTXM15R & $\begin{array}{r}\text { GCC GTC TAA GGC } \\
\text { GAT AAA CA }\end{array}$ & & \\
\hline & TEM F & $\begin{array}{r}\text { ATG AGT ATT CAA } \\
\text { CAT TTC CGTG }\end{array}$ & \multirow[b]{2}{*}{840 bp } & \multirow[t]{2}{*}{$\begin{array}{r}\text { De oliveria } \\
2008\end{array}$} \\
\hline TEM & TEMR & $\begin{array}{r}\text { TTA CCA ATG CTT } \\
\text { AAT CAG TGAG }\end{array}$ & & \\
\hline \multirow[t]{2}{*}{ SHV } & SHVF & $\begin{array}{r}\text { ATG CGT TAT ATT } \\
\text { CGC CTG TG }\end{array}$ & & \multirow{2}{*}{$\begin{array}{r}\text { Schlesinge } \\
\text { r } \text { et al., } \\
2006\end{array}$} \\
\hline & SHVR & $\begin{array}{r}\text { AGA TAA ATC ACC } \\
\text { ACA ATG CGC }\end{array}$ & 776 bp & \\
\hline
\end{tabular}

Table.2 The BLAST results of CTX-M15 E. coli in the gene bank, and compatibility of DNA sequences obtained from NCBI

\begin{tabular}{|c|c|c|c|c|c|}
\hline \multirow[t]{2}{*}{ Gene } & \multicolumn{5}{|c|}{ Nucleotide sequence of query and subject } \\
\hline & Score & Expect value & Identities & Gaps & Accession NO. \\
\hline CTXM15 & $\begin{array}{c}1537 \\
\text { bits(832) }\end{array}$ & 0.0 & 896/915(98\%) & $22 / 915(2 \%)$ & CP034956. \\
\hline CTXM15 & $\begin{array}{c}1607 \\
\operatorname{bits}(870)\end{array}$ & 0.0 & $922 / 944(98 \%)$ & $15 / 944(1 \%)$ & JQ397663.1 \\
\hline \multicolumn{6}{|c|}{ Nucleotide sequence of query and subject } \\
\hline Score & & Expect value & Identities & Gaps & Accession NO \\
\hline 1663 bits $(9$ & & 0.0 & 928/940(99\%) & $8 / 940(0 \%)$ & DQ485310.1 \\
\hline 1607 bits $(8$ & & 0.0 & 915/934(98\%) & $13 / 934(1 \%$ & MG844172 \\
\hline
\end{tabular}


Fig.1 Drugs resistance pattern of ESBL positive and ESBL negative isolates

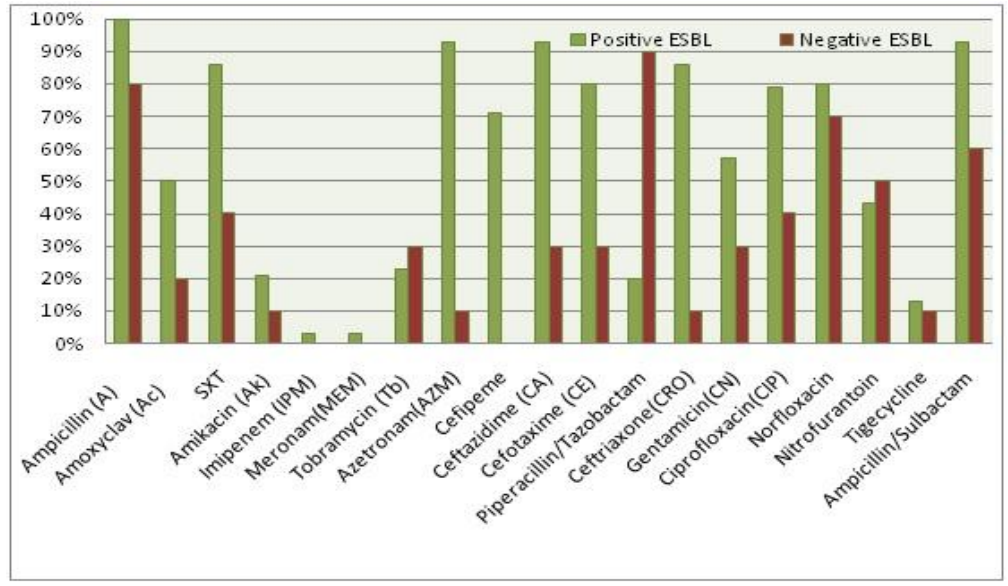

Fig.2 Comparison of confirmatory phenotypic tests for detection of ESBL positive E. coli isolates harboring CTX-M15 gene using PCR as reference method

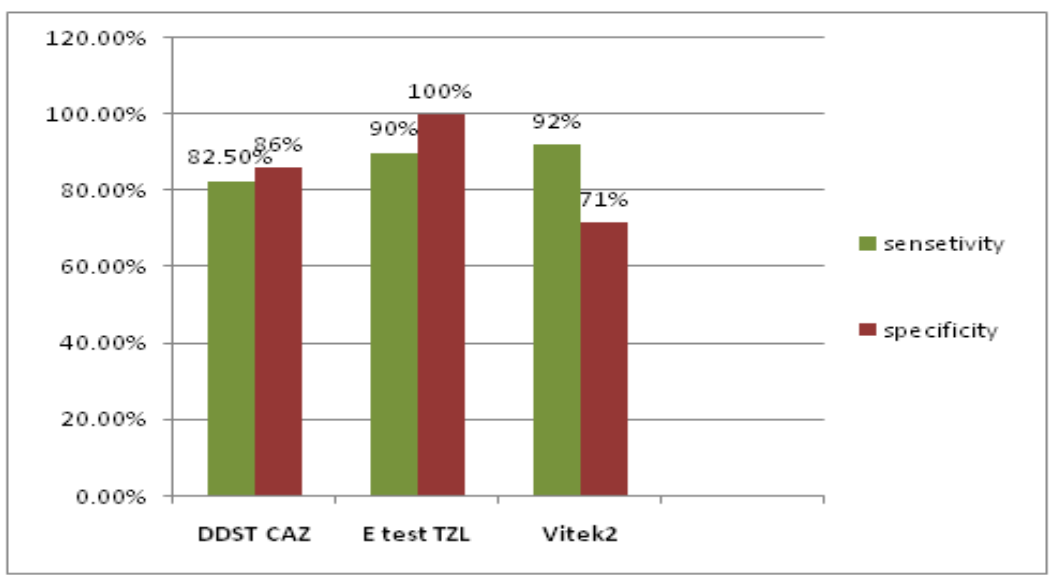

Fig.3 Positive ESBL E. coli using E test ESBL (TZL): Ceftazidime (TZ) / Ceftazidime +clavulanic acid (TZL) shows deformation of the TZ inhibition ellipse indicative of ESBL

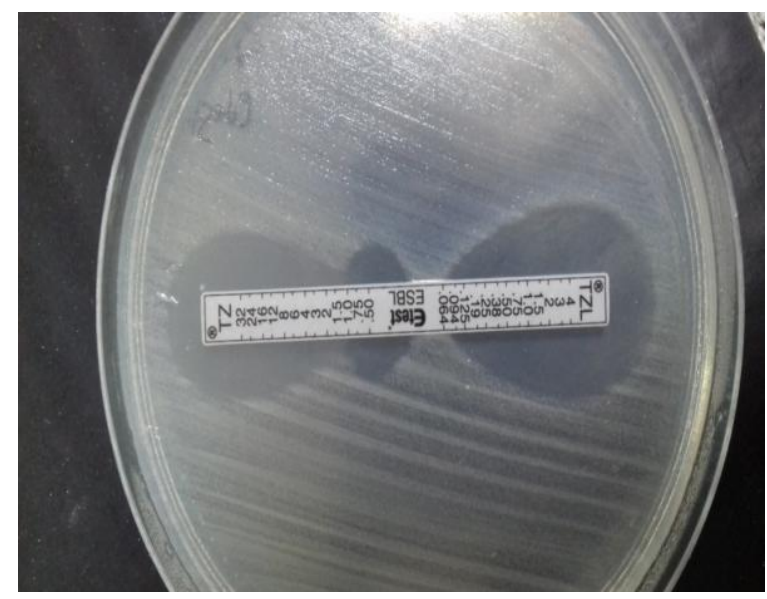


Fig.4 Gel electrophoresis of PCR for detection of CTX-M15 gene

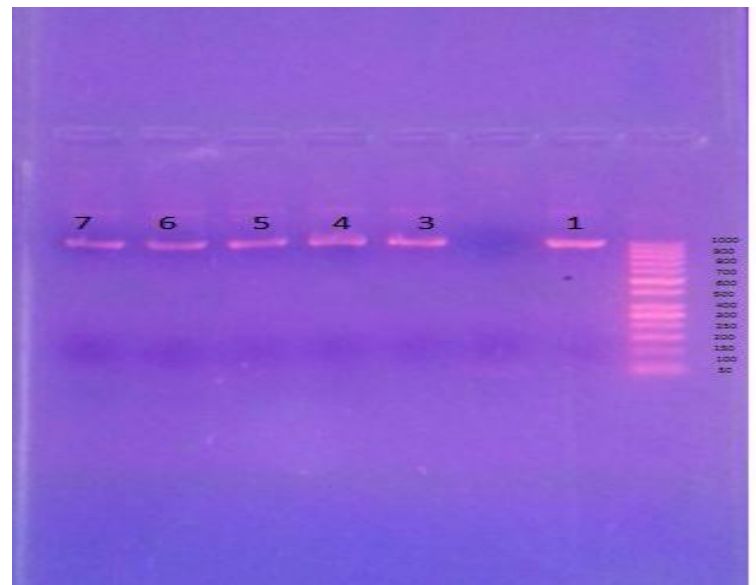

Lane 1: molecular mass marker (100 bp DNA ladder), lane 1, 3, 4, 5, 6 and 7 show positive $E$. coli isolates for CTXM15 gene at $995 \mathrm{bp}$, lane 2 shows negative $E$. coli isolate for CTXM15 gene

Fig.5 Prevalence of ESBL genotyping among studied E. coli isolates

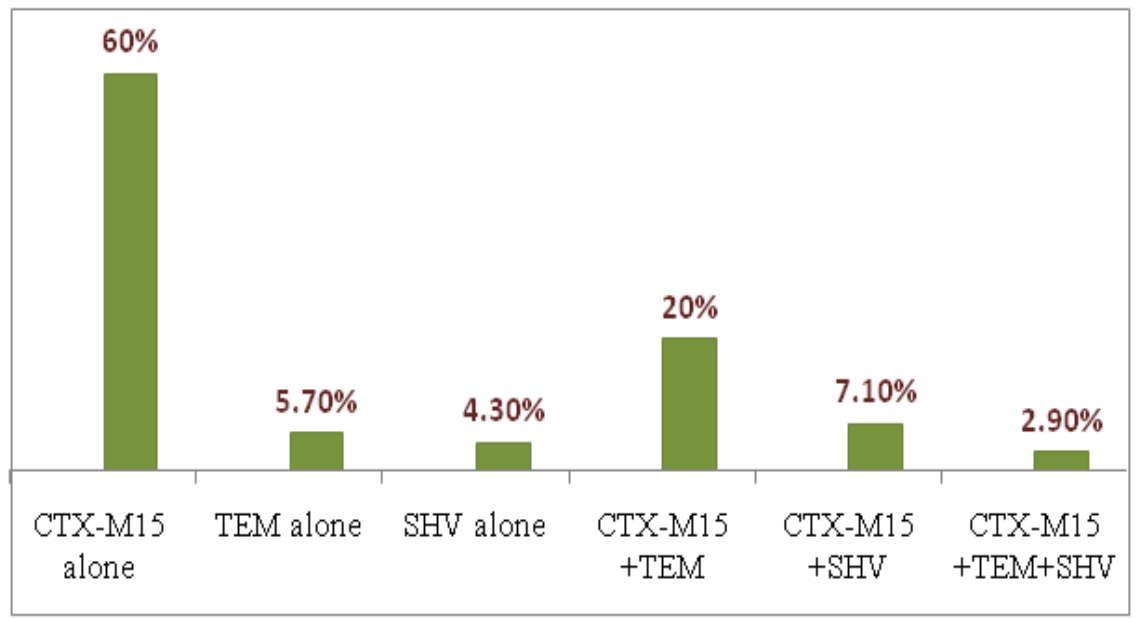

This study had some limitation as relatively small sample size of $80 \mathrm{E}$. coli might be little to obtain significant results; the present study was performed at a single institution in Egypt. Thus, the study results may not reflect the actual prevalence and epidemiology of other geographic areas within the country. Furthermore, epidemiological typing to assess clonality of the isolates was not performed, and this could have added value to the understanding of the epidemiological spread of ESBL genes.
Multicenter molecular based epidemiological studies of ESBL E. coli with longer surveillance duration are recommended for better understanding of the prevalence and distribution of the ESBL genes especially CTX-M15, that can help in prevention of the spread of these resistance bugs in Egyptian hospitals and support the determination of priorities for local intervention actions.

In conclusion, this study highlights the threat of extended spectrum beta lactamases 
producing $E$. coli and their potential multidrug resistance considered a serious problem. CTX-M15 is a predominant gene conferring ESBL-production in $E$. coli clinical isolates causing both hospital- and community-acquired infections in our setting. Early detection of ESBL E. coli strains harboring CTXM-15 is necessary because of uncontrolled dissemination of these plasmid mediated resistant genes. Regarding phenotypic confirmatory tests, Etest (TZL) has higher sensitivity and specificity than other methods (double disc synergy method DDST and Vitek 2) although; Consequently, Vitek 2 system is a reliable semi-automated microbiology system which may be used for routine, accurate and rapid in our clinical settings. Carbapenems are still a good choice treatment option against ESBL E. coli infections.

\section{Conflict of interest}

The authors declare no conflict of interest this research did not receive any specific grant from funding agencies in the public, commercial or not-for- profit sector

\section{Abbreviations}

ESBL: extended spectrum beta lactamases MALDI-TOF MS: Matrix-assisted laser desorption ionization-time of flight mass spectrometry, MDR: multidrug-resistant, ctxm15: cefotaxime hydrolyzing capabilitiesMunich, DDST: double disc synergy, E test: epsilometer test.

\section{References}

Abdallah, H.M., Wintermans, B. B., Reuland, E. A., Koek, A., Al Naiemi, N., Ammar, A. M., and Vandenbroucke-Grauls, C. M. J. E. (2015): Extended-spectrum $\beta$-lactamase-and carbapenemase-producing Enterobacteriaceae isolated from Egyptian patients with suspected blood stream infection. PLoS
One, 10(5), e0128120.

Abdel-Moaty MM, Mohamed WS, Abdel-All SM and El-Hendawy HH. (2016): Prevalence and molecular epidemiology of extended spectrum $\beta$-lactamase producing Escherichia coli from hospital and community settings in Egypt. J App Pharm Sci., 6 (01): 042-047.

Abrar, S., Vajeeha, A., Ul-Ain, N., and Riaz, S. (2017): Distribution of CTX-M group I and group III $\beta$-lactamases produced by Escherichia coli and Klebsiella pneumoniae in Lahore, Pakistan. Microbial pathogenesis, $103,8-12$

Akanbi, B.O., Ojonuba, B. D., and Njoku, R. (2013). Detection of Extended Spectrum [beta]-Lactamase Producing Klebsiella pneumoniae and Escherichia coli in Two Hospitals in the Federal Capital Territory, Abuja, Nigeria. Open Journal of Medical Microbiology, 3(4), 207.

Al-Agamy MH, Seif El-Din MA and Wiegand I. (2006): First description of CTX-M $\beta$ lactamase producing clinical $E$. coli isolates from Egypt. Int J Antimicrob Agents 27: 545-548.

Ali T, ur Rahman S, Zhang L, et al., (2016): ESBL-Producing Escherichia coli from Cows Suffering Mastitis in China Contain Clinical Class 1 Integrons with CTX-M Linked to ISCR1. Front Microbiol; 7. vol. 8, no. 52, pp. 90144-90163, 20

Bajpai T., Pandey M, Varma M and Bhatambare GS. (2017): Prevalence of TEM, SHV, and CTX-M Beta-Lactamase genes in the urinary isolates of a tertiary care hospital. Avicenna J Med; 7: 12-6

Bonnedahl, J., Hernandez, J., Stedt, J., Waldenström, J., Olsen, B., and Drobni, M. (2014). Extended-spectrum $\beta$-lactamases in Escherichia coli and Klebsiella pneumoniae in gulls, Alaska, USA. Emerging infectious diseases, 20(5), 897

Bouxom H., Fournier D, Bouiller K, et al., (2018): Which non-carbapenem antibiotics are active against extended-spectrum $\beta$ lactamase-producing Enterobacteriaceae? Int J Antimicrob Agents.; 52(1): 100

Chakraborty, S., Mohsina, K., Sarker, P. K., Alam, M. D., Karim, M., and Sayem, S. M. (2016). Prevalence, antibiotic susceptibility profiles and ESBL production in Klebsiella 
pneumoniae and Klebsiella oxytoca among hospitalized patients. Periodicum biologorum, 118(1), 53-58.

CLSI. (2018): Performance Standards for Antimicrobial Susceptibility Testing. $28^{\text {th }}$ ed. CLSI supplement M100. Wayne, PA: Clinical and Laboratory Standards Institute

Dasgupta, A., Majumdar, T., and Bhowmik, R. (2018): identification and molecular characterisation of ESBL producing uropathogenic Escherichia coli strains isolated from a tertiary care hospital of Tripura. Journal of evolution of medical and dental sciences-JEMDS, 7(24), 2829-2833.

Djuikoue, I.C., Njajou, O., Kamga, H. G., Fokunang, C., Bongoe, A., Bruno, E. O., and Ngogang, J. (2017): Prevalence of CTX-M beta Lactamases in Escherichia coli from community-acquired urinary tract infections and associated risk factors among women in Cameroon. Journal of Epidemiological Research, 3(1): 51-56.

El-Jade, MR, Parcina M, Schmithausen RM, Stein C, Meilaender A, Hoerauf A, et al., (2016): ESBL Detection: Comparison of Commercially Available Chromogenic Test for Third Generation Cephalosporine Resistance and Automated Susceptibility Testing in Enterobactericeae. PLoS ONE 11(8): e0160203.

Fadil, AA., Hakeem MA and Abdelraheem AR. (2017): ESBL-Producing E. coli and Klebsiella among Patients Treated at Minia University Hospitals. J Infect Dis Preve Med 5: 156.

Fallah, F., Abdolghafoorian, H., Hashemi, A., Goudarzi, H., Gachkar, L., and Hamedany, P. (2016): Antibiotic susceptibility patterns in CTX-M-15-producing Enterobacteraceae isolated from healthy Afghan refugees in Iran. African Journal of Microbiology Research, 10(11), 357-362.

Fam, N.S., and El-Damarawy, M. M. (2008): CTX-M-15 extended spectrum betalactamases detected from intensive care unit of an Egyptian medical research institute. Res J Med Sci, 3(1), 84-91

Fody, A.M., Boubou, L., Moussa, A., Bawa, H. I., Konate, A., Yaou, C., and Traore, A. S. (2017): Phenotypic detection of extended spectrum beta-lactamase in multidrug- resistant Escherichia coli from clinical isolates in Niamey, Niger. African journal of microbiology research, 11(18), 712-717.

Guiral, E., Pons, M. J., Vubil, D., Marí-Almirall, M., Sigaúque, B., Soto, S. M., and Mandomando, I. (2018): Epidemiology and molecular characterization of multidrugresistant Escherichia coli isolates harboring blaCTX-M group 1 extended-spectrum $\beta$ lactamases causing bacteremia and urinary tract infection in Manhiça, Mozambique. Infection and drug resistance, 11, 927.

Harada, S., Ishii Y and Yamaguchi K. (2008): Extended-spectrum beta-lactamases: implications for the clinical laboratory and therapy. Korean J Lab Med.; 28: 401-412.

Hashemizadeh, Z., Kalantar-Neyestanaki, D., and Mansouri, S. (2018): Clonal relationships, antimicrobial susceptibilities, and molecular characterization of extended-spectrum betalactamase-producing Escherichia coli isolates from urinary tract infections and fecal samples in Southeast Iran. Revista da Sociedade Brasileira de Medicina Tropical, 51(1), 44-51.

Kałużna, E., Zalas-Więcek, P., and Gospodarek, E. (2014): Comparison of detection methods for extended-spectrum beta-lactamases in Escherichia coli strains. Postepy Hig Med Dosw, 68, 808-13.

Kanamori, H, Navarro RB, Yano H, Sombrero LT, Capeding MR, Lupisan SP, Olveda RM, Arai K, Kunishima H, Hirakata Y and Kaku M (2011): Molecular characteristics of extended-spectrum beta-lactamases in clinical isolates of Enterobacteriaceae from the Philippines. Acta Trop. 120:140-145.

Khalaf, N.G., Eletreby, M. M. and Hanson, N. D. Characterization of CTX-M ESBLs in Enterobacter cloacae, Escherichia coli and Klebsiella pneumoniae clinical isolates from Cairo, Egypt. BMC Infect. Dis. 9, 84.

Khater, E.S., and Sherif, H. W. (2014): Rapid Detection of Extended Spectrum [Beta]lactamase (ESBL) Producing Strain of Escherichia coli in Urinary Tract Infections Patients in Benha University Hospital, Egypt. British Microbiology Research Journal, 4(4), 443.

Kumar Vergis, J., Pathak, R, M., Sunitha, R., Malik, S. V. S., Barbuddhe, S. B., and 
Rawool, D. B. (2018): A comparative study for detection of extended spectrum $\beta$ lactamase (ESBL) production by Enteroaggregative Escherichia coli (EAEC) strains using double disc, nitrocefin and PCR assays. Journal of microbiological methods, 151, 57-61.

Li, Y., Sun, Q. L., Shen, Y., Zhang, Y., Yang, J. W., Shu, L. B., and Wang, S. (2018). Rapid increase in prevalence of carbapenemresistant enterobacteriaceae (CRE) and emergence of colistin resistance gene mcr-1 in CRE in a hospital in Henan, China. Journal of clinical microbiology, 56(4), e01932-17.

Lim, S. M. S., Wong, P. L., Sulaiman, H., Atiya, N., Shunmugam, R. H., and Liew, S. M. (2019): Clinical prediction models for ESBL-Enterobacteriaceae colonization or infection: A systematic review. Journal of Hospital Infection.

Liu, Y., Heping, X. U., Xiao, M., Yang, Q., and Yingchun, X. U. (2015): Evaluation of the capabilities of disc diffusion and Vitek2compact GN13 methods for testing antimicrobial susceptibility and screening of ESBLs Enterobacteriaceae clinical isolates. double disc synergy test and phenotypic confirmatory disc diffusion test for detection of ESBL production and their antimicrobial resistance patternChinese Journal of Microbiology and Immunology, (2), 139145.

Lopes, R., Cerdeira, L. T., Fernandes, M. R., Pérez-Chaparro, P. J., McCulloch, J. A., and Lincopan, N. (2017): Draft genome sequence of a CTX-M-15-producing endophytic Klebsiella pneumoniae ST198 isolate from commercial lettuce. Journal of global antimicrobial resistance, 10, 19-20.

Maina, D., Revathi, G., and Whitelaw, A. C. (2017): Molecular characterization of multidrug-resistant Klebsiella pneumoniae and Escherichia coli harbouring extended spectrum beta-lactamases and carbapenemases genes at a tertiary hospital, Kenya. Microbiologia Medica, 32(4).

Mashwal, F.A., El Safi, S. H., George, S. K., Adam, A. A., and Jebakumar, A. Z. (2017): Incidence and molecular characterization of the extended spectrum beta lactamaseproducing Escherichia coli isolated from urinary tract infections in Eastern Saudi Arabia. Saudi medical journal, 38(8), 811.

Melo, L.C., Oresco, C., Leigue, L., Netto, H. M., Melville, P. A., Benites, N. R., and Madec, J. Y. (2018). Prevalence and molecular features of ESBL/pAmpC-producing Enterobacteriaceae in healthy and diseased companion animals in Brazil. Veterinary microbiology, 221, 59-66.

Mohajeri, P., Darfarin, G., and Farahani, A. (2014). Genotyping of ESBL producing Uropathogenic Escherichia coli in west of Iran. International journal of microbiology,

Ouedraogo, A. S., Sanou, M., Kissou, A., Sanou, S., Solaré, H., Kaboré, F., and Nacro, B. (2016). High prevalence of extendedspectrum B-lactamase producing enterobacteriaceae among clinical isolates in Burkina Faso. BMC infectious diseases, 16(1), 326.

Pietsch, M., Eller, C., Wendt, C., Holfelder, M., Falgenhauer, L., Fruth, A., and Pfeifer, Y. (2017). Molecular characterisation of extended-spectrum $\beta$-lactamase (ESBL)producing Escherichia coli isolates from hospital and ambulatory patients in Germany. Veterinary microbiology, 200, 130-137.

Ramadan, A.A., Abdelaziz, N. A., Amin, M. A., and Aziz, R. K. (2019). Novel bla CTX-M variants and genotype-phenotype correlations among clinical isolates of extended spectrum beta lactamase-producing Escherichia coli. Scientific reports, 9(1), 4224.

Raut, S., Gokhale, S., and Adhikari, B. (2015). Prevalence of Extended Spectrum BetaLactamases among Escherichia coli and Klebsiella spp isolates in Manipal Teaching Hospital, Pokhara, Nepal. Journal of Microbiology and Infectious Diseases, 5(2).

Rogers, BA., Sidjabat HE. and Paterson DL. (2011): Escherichia coli O25b-ST131: a pandemic, multiresistant, communityassociated strain. J. Antimicrob. Chemother. 66: 1-14.

Strahilevitzm J., Jacoby GA, Hooper DC and Robicsek A (2009): Plasmid-mediated quinolone resistance: a multifaceted threat. 
Clin. Microbiol. Rev. 22:664-689.

Sujatha, R., Kumar, A., and Mishra, V. (2017): A study by double disc diffusion (DDDT) method to compare ceftazidime+ clavulanic acid and cefotaxime+ clavulanic acid for the detection of extended spectrum $\beta$ lactamases among Escherichia coli and Klebsiella pneumoniae in urinary isolates. Int $\mathrm{J}$ Curr Microbiol Appl Sci, 6, 411-5.

Tacão, M., Correia, A., and Henriques, I. (2012): Resistance to broad-spectrum antibiotics in aquatic systems: anthropogenic activities modulate the dissemination of bla CTX-Mlike genes. Appl. Environ. Microbiol., 78(12), 4134-4140.

Tadesse, D.A., Zhao, S., Tong, E., Ayers, S., Singh, A., Bartholomew, M. J., and McDermott, P. F. (2012). Antimicrobial drug resistance in Escherichia coli from humans and food animals, United States, 1950-2002. Emerging infectious diseases, 18(5), 741.

Valenza, G., Nickel, S., Pfeifer, Y., Pietsch, M., Voigtländer, E., Lehner-Reindl, V., and Höller, C. (2017). Prevalence and genetic diversity of extended-spectrum $\beta$-lactamase (ESBL)-producing Escherichia coli in nursing homes in Bavaria, Germany. Veterinary Microbiology, 200, 138-141

Wickramasinghe, N.H., Xu, L., Eustace, A., Shabir, S., Saluja, T., and Hawkey, P. M.
(2012): High community faecal carriage rates of CTX-M ESBL-producing Escherichia coli in a specific population group in Birmingham, UK. Journal of Antimicrobial Chemotherapy, 67(5), 11081113.

Yasir, M., Ajlan, A. M., Shakil, S., Jiman-Fatani, A. A., Almasaudi, S. B., Farman, M., and Ismaeel, N. A. (2018): Molecular characterization, antimicrobial resistance and clinico-bioinformatics approaches to address the problem of extended-spectrum $\beta$-lactamase-producing Escherichia coli in western Saudi Arabia. Scientific reports, 8(1), 14847.

Zhang, J., Zheng, B., Zhao, L., Wei, Z., Ji, J., Li, L., and Xiao, Y. (2014). Nationwide high prevalence of CTX-M and an increase of CTX-M-55 in Escherichia coli isolated from patients with community-onset infections in Chinese county hospitals. BMC infectious diseases, 14(1), 659.

Zhang, Q., Gao, H. Y., Li, D., Li, Z., Qi, S. S., Zheng, S., and Zhang, SH. (2019): Clinical outcome of Escherichia coli bloodstream infection in cancer patients with/without biofilm formation: a single-center retrospective study. Infection and drug resistance, $12,359$.

\section{How to cite this article:}

Rehab M. Ateya, Ghada E. Amr, Hanan E. Mohamed and Neveen M. Gamil. 2019. Molecular Characterization of CTX-M15 Beta Lactamase Producing Escherichia coli Isolates in Intensive Care Units. Int.J.Curr.Microbiol.App.Sci. 8(06): 317-329.

doi: https://doi.org/10.20546/ijcmas.2019.806.036 